\title{
Article
}

\section{Ursodeoxycholic acid protects interstitial Cajal-like cells in the gallbladder from undergoing apoptosis by inhibiting TNF- $\alpha$ expression}

\author{
Jiang-fan WAN ${ }^{1,2}$, Shi-feng $\mathrm{CHU}^{2}$, Xin ZHOU ${ }^{2}$, Yue-ting $\mathrm{Ll}^{3}$, Wen-bin $\mathrm{HE}^{2}$, Feng TAN ${ }^{1}$, Piao $\mathrm{LUO}^{2,4}$, Qi-di Al ${ }^{2,4}$, Qi WANG ${ }^{1}$, \\ Nai-hong $\mathrm{CHEN}^{1,2,4, *}$ \\ ${ }^{1}$ Institute of Clinical Pharmacology, Guangzhou University of Chinese Medicine, Guangzhou 510080, China; ${ }^{2}$ State Key Laboratory of \\ Bioactive Substances and Functions of Natural Medicines, Institute of Materia Medica \& Neuroscience Center, Chinese Academy of \\ Medical Sciences and Peking Union Medical College, Beijing 100050, China; ${ }^{3}$ Beijing Hospital of Integrated Traditional and Western \\ Medicine, Beijing 100039, China; ${ }^{4}$ College of Pharmacy, Hunan University of Chinese Medicine, Changsha 410208, China
}

\begin{abstract}
Hypomotility is a common symptom of gallstone disease, which is accompanied by a loss of interstitial Cajal-like cells (ICLCs) in the gallbladder. Ursodeoxycholic acid (UDCA) is widely used in treating gallstone disease, and has shown anti-apoptotic and antiinflammatory effects apart from its ability to dissolve gallstones. In this study, we investigated the anti-apoptotic and anti-inflammatory effects of UDCA on ICLCs in guinea pigs with gallstones. Guinea pigs were fed a high-cholesterol diet for 8 weeks to induce the formation of gallstones. A group of animals was administered UDCA ( $50 \mathrm{mg} \cdot \mathrm{kg}^{-1} \cdot \mathrm{d}^{-1}$, ig) simultaneously. At the end of 8 weeks, the animals were euthanized with anesthesia, cholecystectomy was performed immediately and gallbladder was collected for further analysis. We showed that in the model group the contractility of gallbladder muscle strips in response to both acetylcholine (ACh) and CCK-8 was severely impaired, which was significantly improved by UDCA administration. Furthermore, UDCA administration significantly reduced the apoptotic ratio of ICLCs, based on the observation of co-localization imaging of apoptotic cells and c-kit-positive cells. Western blotting analysis and real-time PCR results revealed that the TNF- $\alpha /$ Caspase $8 /$ Caspase 3 pathway was suppressed in the UDCA-treated animals, confirming the anti-apoptotic effect of UDCA in the gallbladder. The H\&E staining showed that UDCA administration significantly attenuated inflammatory cell infiltration in the gallbladder wall. In conclusion, UDCA can protect ICLCs in the gallbladder from undergoing apoptosis by inhibiting the TNF- $\alpha /$ Caspase $8 /$ caspase 3 pathway.
\end{abstract}

Keywords: ursodeoxycholic acid; gallstones; interstitial Cajal-like cells

Acta Pharmacologica Sinica (2018) 39: 1493-1500; doi: 10.1038/aps.2017.206; published online 17 May 2018

\section{Introduction}

Gallstones are mainly composed of cholesterol or pigments, and only patients with symptoms or complications are considered to have gallstone disease from a clinical perspective. These symptoms include abdominal pain, cholecystitis or obstructive jaundice. Based on a systematic review of gallstone disease $\mathrm{e}^{[1]}, 10 \%-20 \%$ population of adults have gallstones, and over $20 \%$ of these patients will develop symptoms during their lifetime. Gallbladder hypomotility is an early event in gallstone disease and is presumed to be a "trigger" that promotes stone formation. Dysfunction of gallbladder motility is believed to increase the transit time of supersaturated bile and

\footnotetext{
*To whom correspondence should be addressed.

E-mail chennh@imm.ac.cn

Received 2017-08-21 Accepted 2017-10-31
}

permits cholesterol crystallization and continue growth. Thus, improving gallbladder motility may be an effective strategy to prevent gallstone disease.

Interstitial Cajal-like cells (ICLCs) were found to be distributed throughout the gallbladder and bile duct and play an important role in regulating gallbladder contraction ${ }^{[2,3]}$. As its name implies, ICLCs have similar morphological and immunologic characteristics with the interstitial cells of Cajal (ICCs) that are located in the gastrointestinal tract ${ }^{[4]}$. Both ICLCs and ICCs express c-kit on their cell membranes, providing an ideal biomarker to label these cells. Physiological research has found that ICCs have several important functions in modulating gastrointestinal motility. ICCs can generate and propagate spontaneous electronic slow waves to promote lumen peristalsis and mediate neuronal transmitter signaling from motor neurons to smooth muscle cells ${ }^{[5]}$. Further research has 
found that the ICLCs in the gallbladder may play a similar and important role in gallbladder emptying. A prior study found that patients with cholelithiasis have a significantly lower density of ICLCs in the gallbladder, indicating that the loss of ICLCs may contribute to gallbladder hypomotility and gallstone formation, providing a promising therapeutic target for gallstone disease $\mathrm{e}^{[6-8]}$.

Meanwhile, ursodeoxycholic acid (UDCA) is widely used in treating gallstone disease. Apart from its cholesterolmodulating effect, UDCA has been reported to have antiinflammatory and anti-apoptotic effects ${ }^{[9]}$. Clinical studies have also provided convincing evidence of this finding. UDCA was reported to clinically reduce the incidence of biliary pain and acute cholecystitis in patients with symptomatic gallstones according to an 18-year follow-up study ${ }^{[10]}$. In addition, a diminished number of activated macrophages was accompanied by reduced expression of COX-2 in the gallbladder muscle layer in UDCA-treated patients compared to the placebo-treated group ${ }^{[11]}$. However, whether UDCA can protect ICLCs in gallbladder disease is still unclear. In this present study, we discuss the relationship between ICLCs in the gallbladder and the anti-apoptotic and anti-inflammatory effects of UDCA associated with the underlying mechanism governing motility in the gallbladder.

\section{Materials and methods Animal protocol}

Twenty-one male Hartley guinea pigs (250 to $300 \mathrm{~g}$, purchased from Beijing Vital River Laboratory Animal Technology Co, Ltd, a member of Charles River Laboratories in China. License No: SCXK 2012-0001) were randomly divided into the control, model and UDCA groups after an adaptive feed for $5 \mathrm{~d}$. All animals were given tap water ad libitum and were housed in a 12-h light-dark cycle CV environment. In the control group, regular chow was supplied (cholesterol $<0.01 \%$ ). In the model and UDCA groups, a lithogenic diet containing $2 \%$ cholesterol and $5 \%$ sucrose and $2 \%$ fat (Beijing Huafukang Bioscience Co Inc) was administered. UDCA (Losan Pharma, Germany) was administered daily by gavage with a dose of $50 \mathrm{mg} / \mathrm{kg}$ and, for the control group, an equal volume of solvent was administered. At the end of 8 weeks, all animals were euthanized with anesthesia, and a cholecystectomy was performed immediately after for further analysis (Figure 1A).

This study was carried out in strict accordance with the recommendations in the Guide for the Care and Use of Laboratory Animals. The animal use protocol has been approved by the Institutional Animal Care and Use Committee of Peking Union Medical College.

\section{Immunofluorescence and TUNEL staining}

TUNEL staining was used to detect apoptotic cells, and immunofluorescence for c-kit was utilized to locate ICLCs. Deparaffinized sections were incubated in a citrate buffer solution for $10 \mathrm{~min}$ in a microwave oven for antigen retrieval, and the sample was cooled to room temperature. To increase the penetration of the dyes, the sections were treated with $0.2 \%$
Triton X-100 solution for $10 \mathrm{~min}$, and non-specific binding sites were blocked by $5 \%$ bull serum albumin. The primary antibody (Table 1) was added to the sections in a humidified dark chamber overnight at $4{ }^{\circ} \mathrm{C}$. After rinsing in PBS, the secondary antibody with a 1:250 dilution in PBS (Cy3-labeled goat antirabbit IgG, Beyotime Biotechnology) was incubated at room temperature for $2 \mathrm{~h}$. The TUNEL apoptosis assay kit (Roche Diagnostics, Swissland) was performed according to the manufacturer's instructions. After rinsing in PBS, the sections were examined under a confocal laser scanning microscope (Carl Zeiss LSM800, Germany). The image assay was performed using Image-Pro Plus software (Media Cybernetics, MD, USA).

\section{Contraction response test}

After the gallbladder was removed, gallstone formation was examined with the naked eye, and the total number of gallstones in each group was recorded. To test the contraction response of the gallbladder wall in the presence of excitatory neurotransmitters, muscle strips were longitudinally fixed into $8 \mathrm{~mm}$ in length and $3 \mathrm{~mm}$ in width in an iced PBS solution. After the mucosa layer was carefully removed under a dissecting microscope, the strips were bathed in Kreb's solution containing the following components $(\mathrm{mmol} / \mathrm{L}): \mathrm{NaCl}$ 116.6, $\mathrm{KCl}$ 3.4, $\mathrm{NaHCO}_{3} 21.9, \mathrm{NaH}_{2} \mathrm{PO}_{4} 1.2, \mathrm{CaCl}_{2} 2.5, \mathrm{MgCl}_{2}$ 1.2 and glucose 5.4 and gassed with $95 \% \mathrm{O}_{2}: 5 \% \mathrm{CO}_{2}$ and maintained at $37^{\circ} \mathrm{C}$. The initial resting tension was adjusted to 1 $\mathrm{g}$ during the one-hour equilibration period before different concentrations of CCK-8 or ACh were added into the tanks. In this study, the final concentrations of CCK- 8 ranged from $\log -10 \mathrm{~mol} / \mathrm{L}$ to $\log -6 \mathrm{~mol} / \mathrm{L}$, and ACh ranged from log- 8 $\mathrm{mol} / \mathrm{L}$ to $\log -4 \mathrm{~mol} / \mathrm{L}$. A multichannel transducer record system (BIOPAC Systems Inc, USA) was utilized to monitor the changes in tonic contraction.

\section{Western blotting analysis}

Protein lysates from gallbladder tissues were extracted using the RIPA lysis buffer, and the protein concentrations were measured using a BCA protein assay kit (Beyotime Biotechnology). Samples were run on SDS-polyacrylamide gel electrophoresis and transferred onto PVDF membranes. All unconjugated sites on the membranes were blocked with $3 \%$ BSA in Tris-buffer saline. The membranes were then incubated at $4{ }^{\circ} \mathrm{C}$ overnight with the Bax, Bcl-2, Caspase 8 and Caspase3 primary antibodies. After rinsing with PBST, the membranes were incubated with secondary antibody (HRP-labeled goat antirabbit IgG 1:5000 or HRP-labeled goat anti-mouse 1:5000) for 2 $h$ at room temperature and were then processed for exposure. The densities of the bands were quantified using the protein assay software (Bio-Rad Co, CA, USA). All primary antibodies used in this study are listed in Table 1.

\section{Real-time PCR}

After gallbladder tissues were frozen in liquid nitrogen, the total RNA was extracted by using the Trizol reagent (Invitrogen, CA, USA). Total RNA was then reverse transcribed using the cDNA synthesis kit (Takara Bio Inc, Beijing, China) to 
Table 1. Primary antibodies used in this study.

\begin{tabular}{cccc}
\hline Target & Host & Dilution (usage) & Supplier (Catalog No) \\
\hline c-kit & Rat monoclonal & $1: 250($ IF) & eBioscience (14-1172-82) \\
Bax & Mouse monoclonal & $1: 200(\mathrm{WB})$ & Santa Cruz Biotech (sc-7480) \\
Bcl-2 & Mouse monoclonal & $1: 200(\mathrm{WB})$ & Santa Cruz Biotech (sc-509) \\
Caspase8 & Mouse monoclonal & $1: 200(\mathrm{WB})$ & Santa Cruz Biotech (sc-5263) \\
Caspase3 & Rabbit polyclonal & $1: 1000(\mathrm{WB})$ & Cell Signaling Technology (9662) \\
B-Actin & Mouse monoclonal & $1: 5000(\mathrm{WB})$ & Sigma-Aldrich (A1978) \\
\hline
\end{tabular}

Table 2. Primers used in this study.

\begin{tabular}{ccc}
\hline Gene name & Forward/reverse & Sequence (5'-3') \\
\hline \multirow{2}{*}{ c-kit } & Forward & ATCCTGAAAGTGAGGGCAGC \\
& Reverse & TTGCACCCACGGAACTAGAC \\
SCF & Forward & CTAAGTCCTGAGAAAGGCCGA \\
& Reverse & TCCAAAAGCAAAGCCAATCACA \\
TNF- $\alpha$ & Forward & CCGTCTCCTACCCGGAAAG \\
& Reverse & CGGAATCGGCAAAGTCAAGG \\
GAPDH & Forward & GATTTGGCCGTATTGGACGC \\
& Reverse & GGAACTTGCCGTGGGTAGA \\
\hline
\end{tabular}

obtain cDNA samples according to the manufacturer's instructions. Primers for c-kit and TNF-a and the GAPDH housekeeping gene were synthesized (Tsingke, Beijing, China) and are listed in Table 2. Amplification of cDNA was performed using the qPCR Supermix kit (Transgene Biotech, Beijing, China) and was detected on ABI Prism 7900 (Applied Biosystems, CA, USA). The relative expression levels of mRNAs were calculated using the $\Delta \Delta \mathrm{Ct}$ method with GAPDH as the internal control gene for normalization.

\section{Hematoxylin-eosin (H\&E) staining}

H\&E staining was used to detect inflammatory cell infiltration in the gallbladder wall. The paraffin-embedded tissues were cut at $5 \mu \mathrm{m}$ in thickness using a rotary microtome, and a standard H\&E stain was performed. Slices were examined and captured on a microscope system (Eclipse Ni-U, Nikon Instruments Inc, Tokyo, Japan).

\section{Statistical analysis}

All data were expressed as the mean \pm SD. One-way analysis of variance (ANOVA) was conducted using Prism 5.0 software (GraphPad, San Diego, USA) in all tests, and a $P$ value of 0.05 was considered to be statistically significant.

\section{Results}

\section{General evaluation of gallstone formation}

Gallstones formation was analyzed and calculated after the gallbladders were removed. The bile was muddy and brown, and 6 out of 7 guinea pigs formed gallstones with a total number of 10 in the model group (Figure 1C, 1D). In contrast, no gallstones were observed in the control group. Two out of 7 guinea pigs developed gallstones with a total number of 3 in the UDCA group. The rates of gallstone formation in the control, model, and UDCA groups were $0 \%, 85.7 \%$ and $23.6 \%$, respectively (Figure 1B).

\section{UDCA inhibits apoptosis in ICLCs}

Sections were obtained from 3 individual animals per group. For each section, 5 random fields were chosen. In general, most c-kit-positive cells were distributed in the muscular layer and had fusiform to oval shapes (Figure 2A). We calculated the number of c-kit-positive cells and dual-positive cells in cross-sections. There were fewer c-kit-positive cells that were observed in the model group ( $P<0.05$ vs control group), and a slight increase was detected in the UDCA group, but the findings did not reach statistical significance (Figure $2 \mathrm{~B}$ ). The ratio of dual-positive cells to c-kit-positive cells represented the apoptotic ratio in ICLCs. The result showed a significantly increased apoptotic ratio in ICLCs in the model group $(P<0.01$ vs control group). However, in the UDCA group, the ratio was significantly reduced $(P<0.01$ vs model group) (Figure $2 \mathrm{C}$ ).

\section{UDCA ameliorates the tonic contraction response}

All muscle strips showed a dose-dependent increase in tonic contraction in response to both CCK-8 and ACh, but this finding was remarkably reduced in the model group $(P<0.01$ or $P<0.001$, control vs model). Of note, the tonic contraction in the UDCA group was restored at every concentration of ACh and CCK-8 $(P<0.05$ or $P<0.01$, model vs UDCA) (Figure 3$)$.

\section{UDCA inhibits the TNF- $\alpha /$ Caspase $8 /$ Caspase 3 pathway}

Western blotting analyses showed no expression of Bax in the control group. Bax was notably increased in the model group and down-regulated in the UDCA group. The expression of $\mathrm{Bcl}-2$ did not change among the different groups (Figure 4A). As a result, the ratio of Bax to Bcl-2 in the model group and in the UDCA group was increased by $2.54 \pm 0.45$ and $1.27 \pm 0.25$ fold, respectively, compared to the control group (Figure 4D). The expression of cleaved Caspase 8 and its downstream effector, cleaved Caspase3, were significantly elevated in the model group $(P<0.01$, model vs control), while there was reduced expression of these proteins in the UDCA group $(P<0.01$, UDCA vs model) (Figure 4B and 4C). Real-time PCR results showed that the mRNA expression level of TNF-a was signifi- 
A

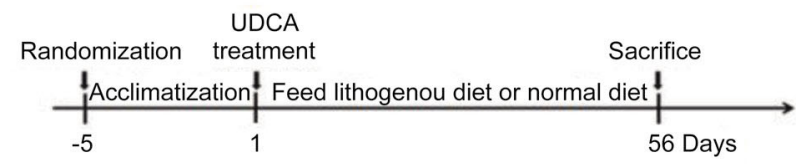

C

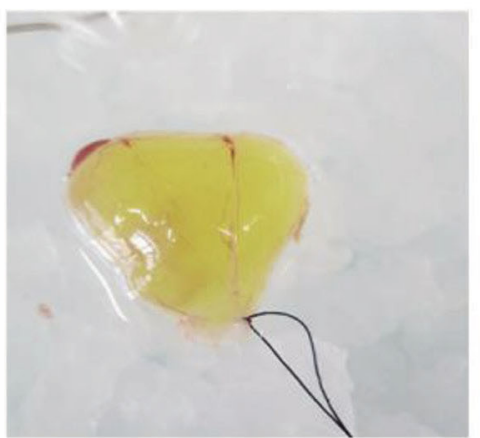

B

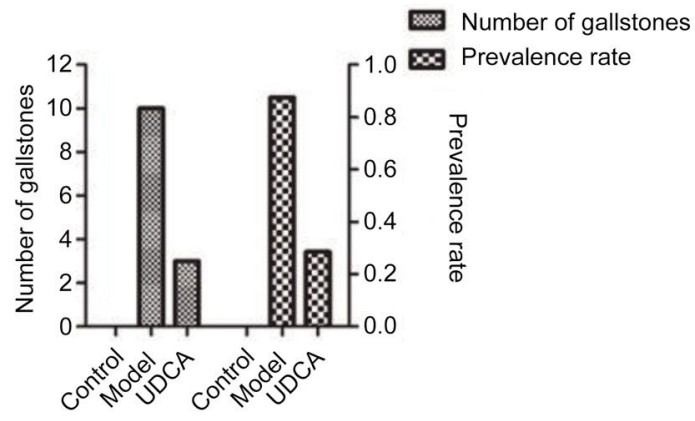

D

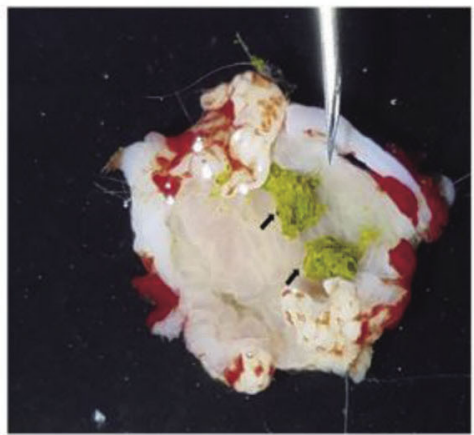

Figure 1. (A) The diagram of feeding animals. (B) Left $Y$ axis represents the total number of gallstones in each group, and right $Y$ axis means the prevalence rate of gallstone disease in each group. (C) In model group, the bile is muddy and brown. (D) The gallstones are obvious in model group (black arrows shown).

A
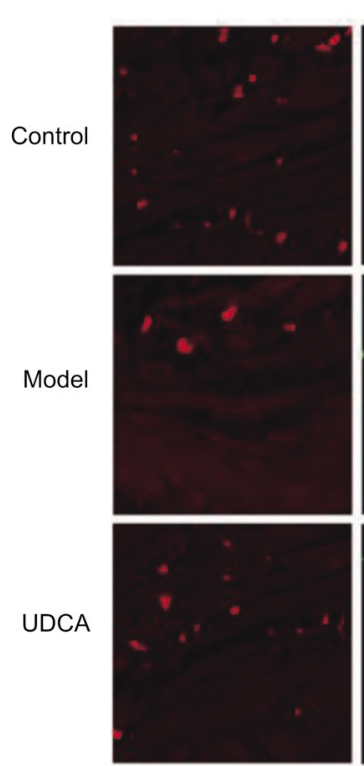

TUNEL
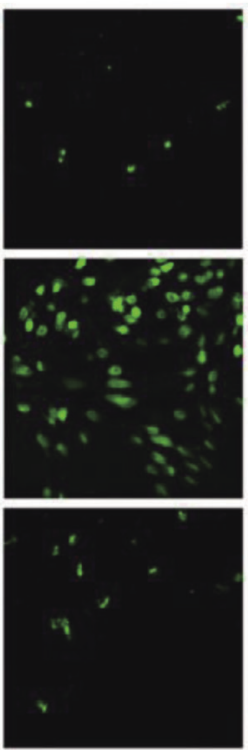

Merge
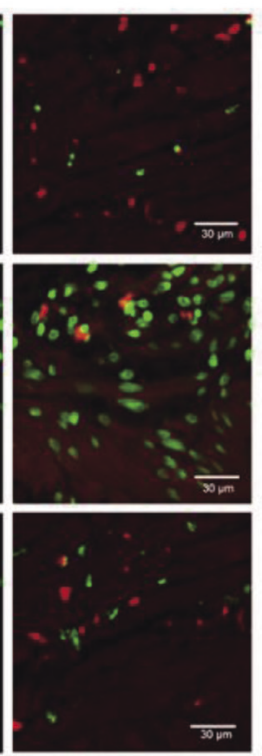

B

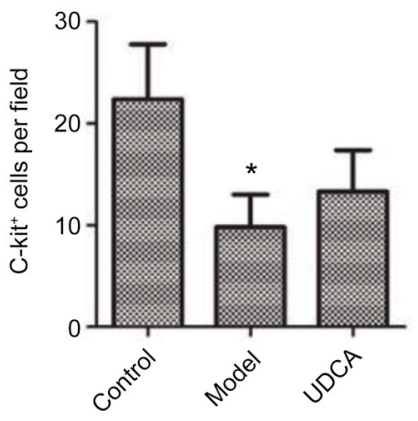

C

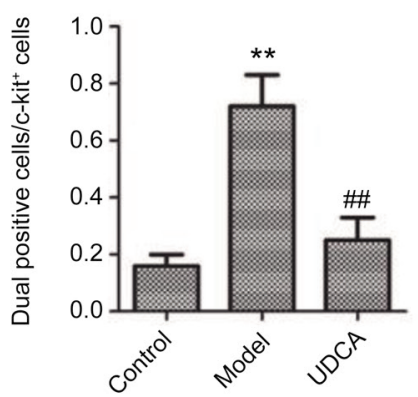

Figure 2. (A) The representative co-localization images of control group, model group and UDCA group. (B) The number of c-kit positive cells in a single field in different groups. (C) The ratio of dual positive cells to c-kit positive cells in different groups. ${ }^{*} P<0.05,{ }^{* *} P<0.01$, control vs model; ${ }^{\# \#} P<0.01$, model vs UDCA.

cantly increased in the model group $(P<0.001$, model vs vehicle control), whereas the levels returned to baseline in the UDCA group $(P<0.001$, UDCA vs model) (Figure $4 \mathrm{E})$.

\section{UDCA reduces SCF/c-kit signaling}

The high-cholesterol diet significantly down-regulated the mRNA expression of c-kit and SCF in the gallbladder wall 
A

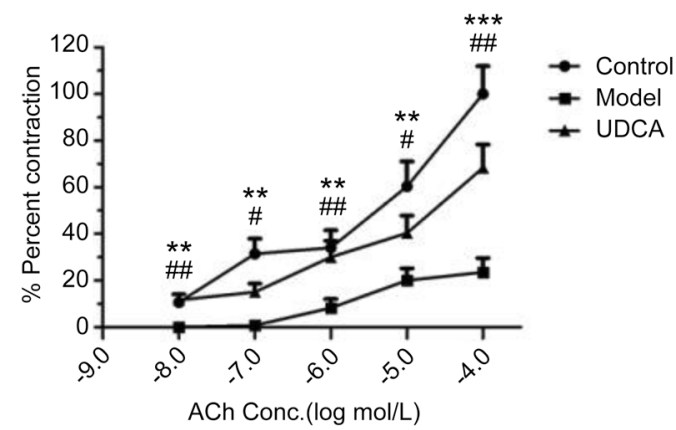

$\mathrm{B}$

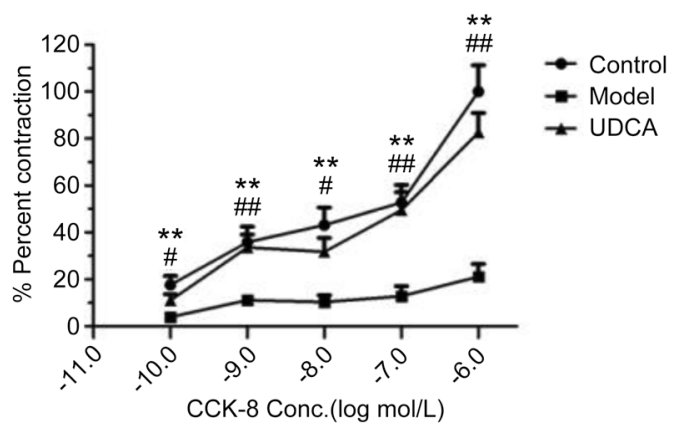

Figure 3. (A) The percent contraction of muscle strips at different concentrations of ACh. (B) The percent contraction of muscle strips at different concentrations of CCK-8. ${ }^{* *} P<0.01,{ }^{* * *} P<0.001$, control vs model; ${ }^{\#} P<0.05,{ }^{\# \#} P<0.01$, UDCA vs model.

A

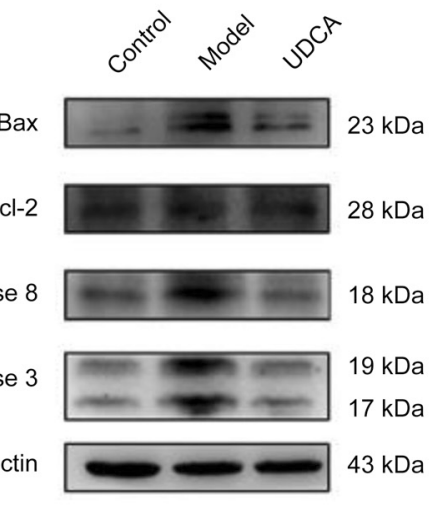

C

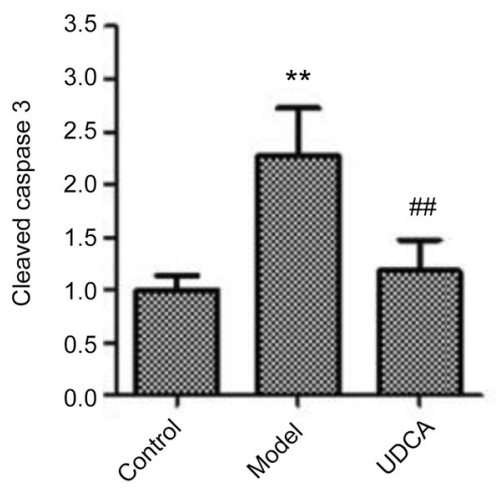

E

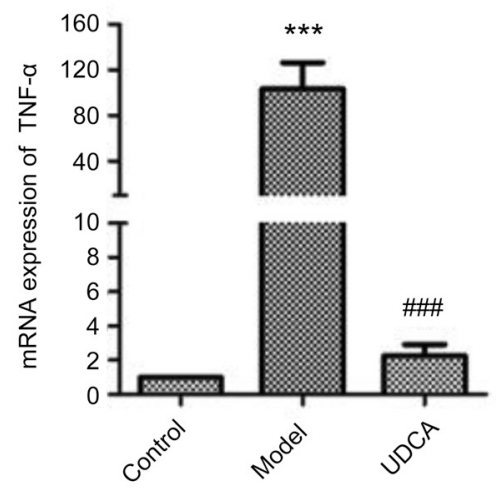

B

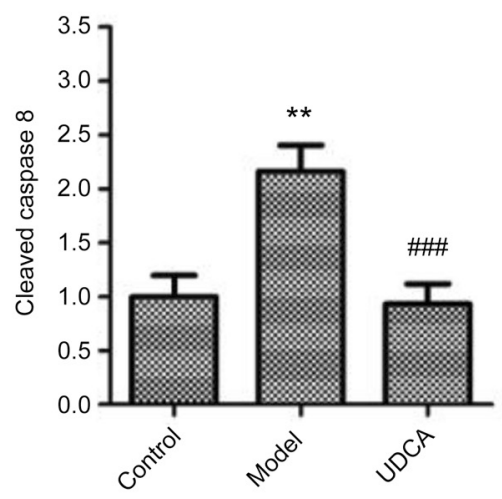

D

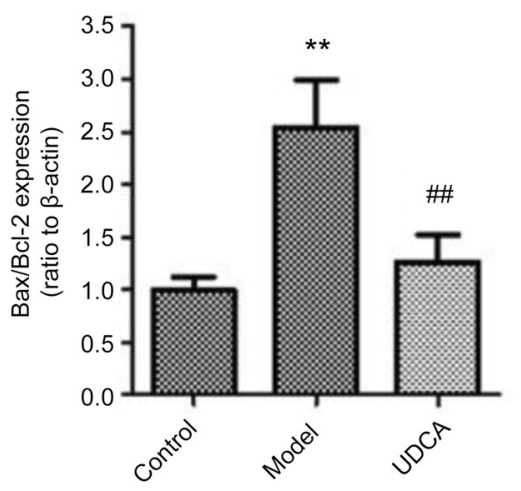

Figure 4. (A) The representative band images of Western blot. (B) Quantification of relative expression of cleaved Caspase 8. (C) Quantification of relative expression of cleaved Caspase 3. (D) Quantification of the intensities of Bax and $\mathrm{Bcl}-2$ and expressed as $\mathrm{Bax} / \mathrm{Bacl}-2$ ratio. (E) The relative mRNA expression of TNF- $\alpha .{ }^{* *} P<0.01,{ }^{* * *} P<0.001$, control vs model; ${ }^{\# \#} P<0.01$, ${ }^{\# \#} P<0.001$, UDCA vs model. 
when compared to the control group $(P<0.05$ for c-kit; $P<0.01$ for SCF). UDCA ameliorated the mRNA expression of c-kit $(P<0.05$, UDCA $v$ s model) but did not have the same effect on SCF (Figure 5).

\section{UDCA prevents inflammatory cells infiltration}

The H\&E staining showed a relative severity of inflammation in the model group. Specifically, when compared to the control group, more inflammatory cells infiltrated the muscular layer and the mucous layer in the model group. However, UDCA had an anti-inflammatory effect on the gallbladder wall and reduced the number of inflammatory cells in the muscular and mucous layers of the gallbladder (Figure 6).

\section{Discussion}

Interstitial cells of Cajal (ICCs) in the GI tract were first described over 100 years ago by the Spanish neuroscientist Cajal $^{[12]}$. Later, physiologists found other types of interstitial cells with morphological and immunologic characteristics similar to those of ICCs and named them interstitial Cajallike cells (ICLCs). These cells are distributed in a variety of locations. Most are located in the cavitary organs, including the bladder, male and female reproductive systems, gallbladder and bile ducts, urinary tract, pancreas, and even in the heart (at the junction between the pulmonary vein and the left atrium), and reports with new findings are still ongoing ${ }^{[13-17]}$.
Today, ICCs and ICLCs are known as the pacemaker cells that generate and propagate slow wave potentials to promote smooth muscle contraction ${ }^{[18]}$. A lack of ICCs is now becoming a well-known pathology that underlies the motor dysfunction in the GI tract. In several studies conducted on ICCs, ultrastructural images showed either smooth muscle cells and ICCs or the ICCs itself that were electronically coupled by forming gap junctions, and close contact (less than $20 \mathrm{~nm}$ ) between the ICCs and the varicosities of motor neurons were found. In the $\mathrm{W} / \mathrm{W}^{\mathrm{V}} \mathrm{c}$-kit mutant mice, the slow wave potentials are absent, and the postjunctional response to stimulation of enteric motor neurons was defective. Additionally, other studies have found excitatory and inhibitory receptors expressed in the membranes of ICCs, indicating the possible role of ICCs in neurotransmission ${ }^{[5]}$. SCF/c-kit signaling is vital to ICCs and was highlighted in research on ICCs. c-kit is a type of tyrosine kinase receptor that is located in the membranes of ICCs and regulates several important proteins, such as phosphatidylinositol 3'-kinase (PI3-kinase), phospholipase C- $\gamma$ (PLC- $\gamma$ ), phospholipase D, SRC family kinases, p21ras GTPase-activating protein and mitogen-activated protein kinase (MAPK) ${ }^{[19]}$.

Although studies in this field mainly focus on the ICCs in the GI tract, several in vitro studies have discussed the importance of ICLCs in the gallbladder. Morphological and physiological studies have confirmed the existence of ICLCs in the gallbladder in human and mouse and guinea pigs ${ }^{[2,20,21]}$, and the loss of ICLCs in the gallbladder is related to a hypomo-
A

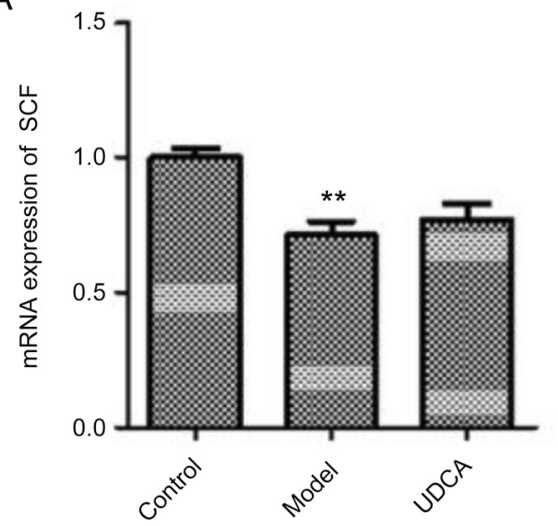

B

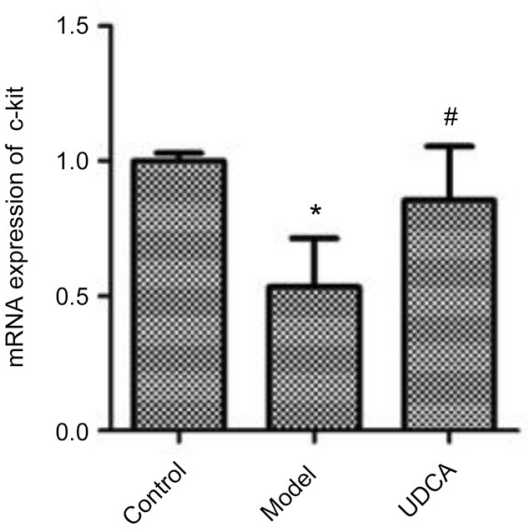

Figure 5. The relative mRNA expression of SCF (A) and its ligand c-kit (B). ${ }^{*} P<0.05,{ }^{* *} P<0.01$, control vs model; ${ }^{\#} P<0.05$, UDCA vs model.
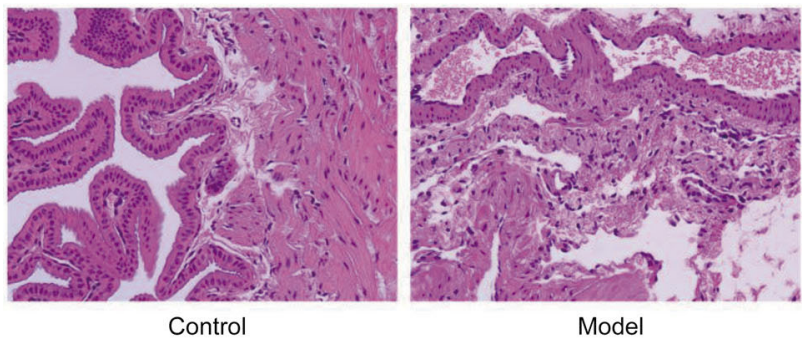

Model

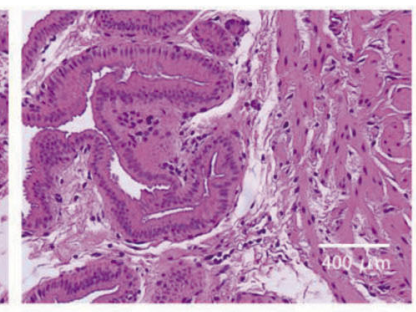

UDCA

Figure 6. The representative images of $\mathrm{H} \& \mathrm{E}$ staining of gallbladder wall. 


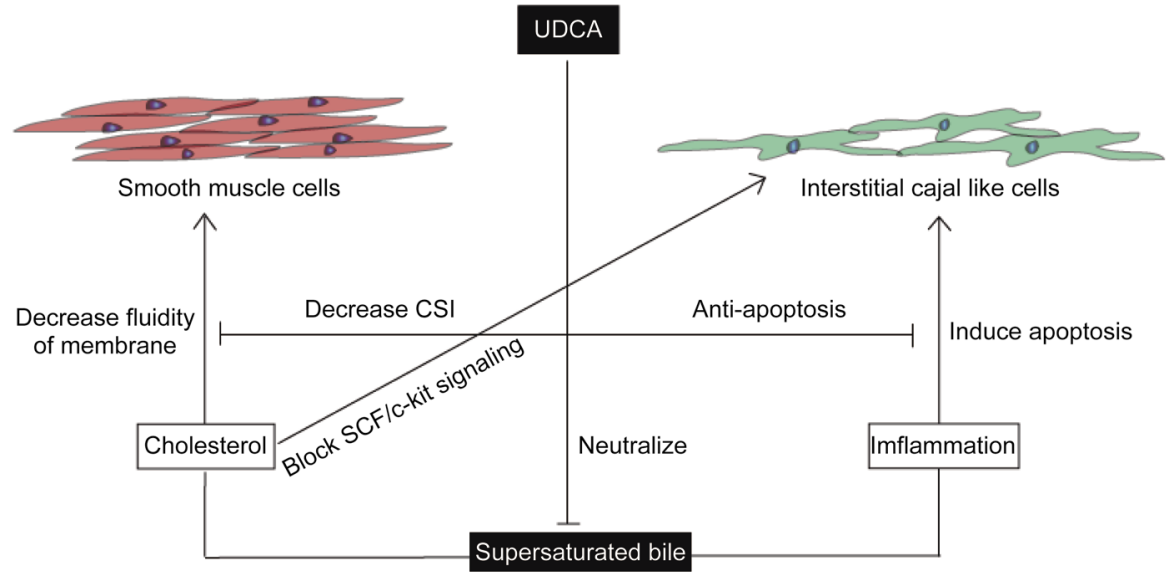

Figure 7. The overall effect of UDCA in improving contractility of gallbladder.

tility disorder ${ }^{[6]}$. However, the functional role of ICLCs in the gallbladder is still rather unclear. A study reported that the CCK-A receptor is expressed in ICLCs. The contraction response of ICLCs-deprived muscle strips was significantly reduced in response to exogenous CCK-8, implying that the ICLCs in the gallbladder could mediate the excitatory input of $\mathrm{CCK}^{[22]}$. However, whether ICLCs also mediate other neurotransmitters related to gallbladder emptying, such as acetylcholine, adrenaline, substance $\mathrm{P}$ or vasoactive intestinal peptide, is unknown. In this study, we found the contraction response of gallbladder strips to ACh was largely suppressed in the model group. Therefore, in future studies, determining whether ICLCs specifically removed from the gallbladder exert an impaired contraction to $\mathrm{ACh}$, similar to what has been confirmed in the GI tract, will be invaluable ${ }^{[23]}$.

The causes of ICLC loss can be complicated, as inflammation can be a key factor that leads to the apoptosis of ICLCs. An in vitro study found that the apoptotic rate of ICLCs significant increased after co-culture with isolated neutrophils from guinea pigs that were subjected to bile duct ligation ${ }^{[24]}$. In the present study, we observed similar results in which an increased number of inflammatory cells infiltrated the tissue in the model group. The expression of c-kit was down-regulated, and the loss of ICLCs may be due to the activation of the TNF-a/Caspase8/Caspase3 pathway. During this process, TNF-a, a proinflammatory cytokine and an apoptosis-inducing factor, can bind to its TNFR1 receptor and cleave proCaspase 8 into activated Caspase 8 and activate pro-Caspase3 into activated Caspase3, which is the final executioner Caspase that leads to cell death. The ratio of Bax to Bcl-2 can be a reliable indicator to monitor cell apoptosis. An increased Bax to Bcl-2 ratio suggests that apoptosis has been initiated, while a reduced ratio suggests that apoptosis has been inhibited.

UDCA has been used clinically to dissolve stones and to protect the liver by inhibiting cholesterol absorption from the intestine and prevent cholesterol secretion into bile. UDCA also functions to neutralize toxic hydrophobic bile acids. A previous study found that, after treatment with
UDCA for 4 weeks, the gallbladder muscle cells isolated from patients with cholesterol gallstones had significantly lower levels of oxidative stress and inflammation and restored the contractility of smooth muscle cells as well ${ }^{[25]}$. Another study found a diminished number of activated macrophages infiltrated in the muscular layer of the gallbladder in patients with cholesterol gallstones after treatment with UDCA for 30 days $^{[11]}$. In the present study, we also show that UDCA effectively prevents the infiltration of inflammatory cells in the gallbladder wall in guinea pigs fed a high-cholesterol diet. All of these findings indicate that UDCA exerts anti-inflammatory effects that are independent of their ability to dissolve or neutralize gallstones. The glucocorticoid-like structure of UDCA may contribute to this anti-inflammatory effect, as UDCA has been reported to bind to the glucocorticoid nuclear receptor and induce mild activation ${ }^{[26]}$.

In the present study, we describe the anti-apoptotic effect of UDCA on ICLCs that may partially contribute to the improvement of gallbladder motility. However, the pathogenesis of motor deficits may include multiple factors. Here, we propose a hypothesis to explain the pathogenic role of supersaturated bile and the therapeutic role of UDCA. First, UDCA can decrease cholesterol concentration, bile viscosity and sedimentable fractions, which can be represented by a lower cholesterol saturation index $(\mathrm{CSI})^{[27]}$. High levels of cholesterol in supersaturated bile can infiltrate through the epithelial cells and convert to cholesteryl esters, which decreases the fluidity of the smooth muscle cell membrane $e^{[28]}$ and blocks cholecystokinin 1 receptor signaling ${ }^{[29]}$. Meanwhile, SCF/c-kit signaling is indispensable to maintain the normal function of pace-making and the survival of ICLCs ${ }^{[30]}$. The cholesterol-scavenge effect of UDCA from the cell membrane may thus enhance the robustness of the network of ICLCs. Lastly, supersaturated bile and hydrophobic bile acids can promote inflammation in the gallbladder, and the proinflammatory TNF-a cytokine that is released can trigger the TNF-a/Caspase8/Caspase3 cascade to induce cell apoptosis. We show in this study that 
UDCA reduces the level of TNF- $\alpha$ and inflammatory cell infiltration and further prolongs ICLCs survival by inhibiting this pathway. Taken together, our findings provide a new perspective on understanding the comprehensive effect of UDCA on gallstone disease.

\section{Acknowledgements}

This work was supported by the National Natural Science Foundation of China (81473570, U1402221, 81560663, and 81560685), Beijing Key Laboratory of New Drug Mechanisms and Pharmacological Evaluation Study (BZ0150), CAMS Innovation Fund for Medical Sciences (CIFMS) (2016-I2M1-004), the Scientific Research Foundation of the Higher Education Institutions of Hunan Province (15K091), State Key Laboratory Fund Open Project (GTZK201610), and China Postdoctoral Science Foundation (2013M540066).

\section{Author contribution}

Jiang-fan WAN designed and carried out the experiments and drafted the manuscript; Shi-feng $\mathrm{CHU}$ and Feng TAN provided advice on the experimental design; Xin ZHOU, Qi-di AI and Piao LUO participated in the animal experiments; Wenbin HE helped with data analysis and with the graphic software; Qi WANG checked and modified the draft; Nai-hong CHEN and Yue-ting LI provided all the funds. All authors read and approved the final manuscript.

\section{References}

1 Lammert F, Gurusamy K, Ko CW, Miquel JF, Méndez-Sánchez N, Portincasa P, et al. Gallstones. Nat Rev Dis Primers 2016; 2: 1-7.

2 Pasternak A, Gajda M, Gil K, Matyja A, Tomaszewski KA, Walocha JA, et al. Evidence of interstitial Cajal-like cells in human gallbladder. Folia Histochem Cytobiol 2012; 50: 581-5.

3 Ahmadi O, Nicholson Mde L, Gould ML, Mitchell A, Stringer MD. Interstitial cells of Cajal are present in human extrahepatic bile ducts. J Gastroenterol Hepatol 2010; 25: 277-85.

4 Hinescu ME, Ardeleanu C, Gherghiceanu M, Popescu LM. Interstitial Cajal-like cells in human gallbladder. J Mol Histol 2007; 38: 275-84.

5 Sanders KM, Ward SM, Koh SD. Interstitial cells: regulators of smooth muscle function. Physiol Rev 2014; 94: 859-907.

6 Pasternak A, Gil K, Gajda M, Tomaszewski KA, Matyja A, Walocha JA. Interstitial cajal-like cell: a new player in cholelithiasis? Am J Gastroenterol 2014; 109: 603-4.

7 Franks I. Gallbladder: Loss of interstitial Cajal-like cells in the gallbladder might contribute to gallstone formation. Nat Rev Gastroenterol Hepatol 2012; 9: 689.

8 Pasternak A, Gil K, Matyja A, Gajda M, Sztefko K, Walocha JA, et al. Loss of gallbladder interstitial Cajal-like cells in patients with cholelithiasis. Neurogastroenterol Motil 2013; 25: e17-24.

9 Bellentani S. Immunomodulating and anti-apoptotic action of ursodeoxycholic acid: where are we and where should we go? Eur J Gastroenterol Hepatol 2005; 17: 137-40.

10 Tomida S, Abei M, Yamaguchi T, Matsuzaki Y, Shoda J, Tanaka N, et al. Long-term ursodeoxycholic acid therapy is associated with reduced risk of biliary pain and acute cholecystitis in patients with gallbladder stones: a cohort analysis. Hepatology 1999; 30: 6-13.

11 Guarino MP, Carotti S, Morini S, Perrone G, Behar J, Altomare A, et al.
Decreased number of activated macrophages in gallbladder muscle layer of cholesterol gallstone patients following ursodeoxycholic acid. Gut 2008; 57: 1740-1.

12 Cajal SR. Histologie du systeme Nerveux de l'Homme et des vertebres. Pairs: Maloine, 1911.

13 Marín VG, Freire MA. The interstitial cells of Cajal in pancreas. J Cell Mol Med 2005; 9: 475.

14 Wickramasinghe SR, Patel VV. Local innervation and atrial fibrillation. Circulation 2013; 128: 1566-75.

15 Drumm BT, Koh SD, Andersson KE, Ward SM. Calcium signalling in Cajal-like interstitial cells of the lower urinary tract. Nat Rev Urol 2014; 11: 555-64.

16 Popescu LM, Ciontea SM, Cretoiu D. Interstitial Cajal-like cells in human uterus and fallopian tube. Ann N Y Acad Sci 2007; 1101: 139-65.

17 Huang Y, Mei F, Yu B, Zhang HJ, Han J, Jiang ZY, et al. Distribution of the interstitial Cajal-like cells in the gallbladder and extrahepatic biliary duct of the guinea-pig. Acta Histochem 2009; 111: 157-65.

18 Balemba OB, Bartoo AC, Nelson MT, Mawe GM. Role of mitochondria in spontaneous rhythmic activity and intracellular calcium waves in the guinea pig gallbladder smooth muscle. Am J Physiol Gastrointest Liver Physiol 2008; 294: G467-76.

19 Lennartsson J, Ronnstrand L. Stem cell factor receptor/c-Kit: from basic science to clinical implications. Physiol Rev 2012; 92: 1619-49.

20 Lavoie B, Balemba OB, Nelson MT, Ward SM, Mawe GM. Morphological and physiological evidence for interstitial cell of Cajal-like cells in the guinea pig gallbladder. J Physiol 2007; 579: 487-501.

21 Sun X, Yu B, Xu L, Dong W, Luo H. Interstitial cells of Cajal in the murine gallbladder. Scand J Gastroenterol 2006; 41: 1218-26.

22 Xu D, Yu BP, Luo HS, Chen LD. Control of gallbladder contractions by cholecystokinin through cholecystokinin-A receptors on gallbladder interstitial cells of Cajal. World J Gastroenterol 2008; 14: 2882-7.

23 Ward SM, Beckett EA, Wang X, Baker F, Khoyi M, Sanders KM. Interstitial cells of Cajal mediate cholinergic neurotransmission from enteric motor neurons. J Neurosci 2000; 20: 1393-403.

24 Huang ZP, Qiu H, Yang Y, Yu BP. Effect of neutrophils on gallbladder interstitial Cajal-like cells in guinea pig model of acute cholecystitis. Cell Physiol Biochem 2016; 39: 2033-43.

25 Guarino MP, Cong P, Cicala M, Alloni R, Carotti S, Behar J. Ursodeoxycholic acid improves muscle contractility and inflammation in symptomatic gallbladders with cholesterol gallstones. Gut 2007; 56: 815-20.

26 Weitzel C, Stark D, Kullmann F, Schölmerich J, Holstege A, Falk W. Ursodeoxycholic acid induced activation of the glucocorticoid receptor in primary rat hepatocytes. Eur J Gastroenterol Hepatol 2005; 17: 169-77.

27 Fischer S, Müller I, Zündt BZ, Jüngst C, Meyer G, Jüngst D. Ursodeoxycholic acid decreases viscosity and sedimentable fractions of gallbladder bile in patients with cholesterol gallstones. Eur J Gastroenterol Hepatol 2004; 16: 305-11.

28 Xiao ZL, Amaral J, Biancani P, Behar J. Impaired cytoprotective function of muscle in human gallbladders with cholesterol stones. Am J Physiol Gastrointest Liver Physiol 2005; 288: G525-32.

29 Wang DQ, Schmitz F, Kopin AS, Carey MC. Targeted disruption of the murine cholecystokinin-1 receptor promotes intestinal cholesterol absorption and susceptibility to cholesterol cholelithiasis. J Clin Invest 2004; 114: 521-8.

30 Torihashi S, Nishi K, Tokutomi Y, Nishi T, Ward S, Sanders KM. Blockade of kit signaling induces transdifferentiation of interstitial cells of cajal to a smooth muscle phenotype. Gastroenterology 1999; 117: $140-8$. 